\title{
Experiencia de un Ciclo de Mejora en el Aula en el ámbito del marketing financiero
}

\section{Experience of an Improvement Cycle in Classroom in the field of financial marketing}

ISABEL MARÍA ROSA DÍAZ

ORCID: https://orcid.org/0000-0001-9138-289X

Universidad de Sevilla. Departamento de

Administración de Empresas y Marketing

imrosa@us.es

Fecha de recepción: 02-06-2019

Fecha de aceptación: 10-06-2019

DOI: http://dx.doi.org/10.12795/9788447221912.013

Pp.: 313-335 


\section{Resumen}

El interés por incorporar en mi docencia habitual las premisas de la innovación docente, en la que los estudiantes adoptan un rol activo y conductor de su propio aprendizaje, me ha llevado a aplicar un $\mathrm{Ci}$ clo de Mejora en el Aula (CIMA) en el marco de la asignatura Marketing Financiero, perteneciente al Grado en Finanzas y Contabilidad de la Universidad de Sevilla. En el presente trabajo se presenta el diseño del mismo, así como las principales dificultades experimentadas y los logros más destacados. Las actividades docentes diseñadas para alcanzar los objetivos planteados han sido diversas, y han tomado como base tanto el trabajo individual como de equipos de trabajo de los estudiantes, las clases generales, los estudios de casos, los debates y consultorías interactivas y la presentación escrita y oral de informes. En cuanto a la valoración de resultados, se han utilizado gráficos y escaleras de aprendizaje.

Palabras clave: Marketing Financiero; Grado en Finanzas y Contabilidad; Docencia universitaria; Experimentación docente universitaria; Escaleras de aprendizaje.

\section{Abstract}

My interest in incorporating the premises of teaching innovation into my usual teaching, in which students adopt an active role and lead their own learning, has led me to apply an Improvement Cycle in Classroom (ICIC) within the framework of the subject Financial Marketing, belonging to the Degree in Finance and Accounting of the University of Seville. The present work presents its design, as well as the main dificulties experienced and the main achievements. The teaching activities designed to achieve the objectives have been diverse, and have been based on both individual work and students work teams, general lessons, case studies, debates and interactive consultancies and written and oral presentation of reports. In terms of evaluating results, graphs and learning ladders have been used.

Keywords: Financial Marketing; Degree in Finance and Accounting; University teaching; University teaching experimentation; Learning ladders. 


\title{
Contexto en el que se desarrolla la asignatura Marketing Financiero
}

El Grado en Finanzas y Contabilidad de la Universidad de Sevilla se imparte en la Facultad de Turismo y Finanzas desde el curso 2009-2010, y se sitúa dentro de la Rama de Conocimiento de Ciencias Sociales y Jurídicas. Dentro del mencionado Grado, la asignatura Marketing Financiero tiene carácter optativo y se sitúa en el 4 으 curso, 2o cuatrimestre, contando con 74 estudiantes matriculados en el curso 2018-2019. Los contenidos de la asignatura están estructurados en tres partes: 1a. Conceptos básicos de marketing relacional y estrategias (temas 1 y 2); 2a. Gestión de servicios financieros (temas 3, 4 y 5); 3a. El sector bancario (temas 6 y 7 ).

\section{Diseño previo del Ciclo de Mejora en el Aula}

\author{
Los contenidos y el mapa de \\ contenidos y problemas
}

El tema seleccionado para aplicar el Ciclo de Mejora en el Aula (CIMA) se centra en la gestión comercial de servicios financieros (tema 4) y la creación de valor para el cliente (tema 5). Así, en el mapa de contenidos y problemas a parecen conceptos estructuradores de ambos temas (Figura 1).

Jornadas de Formación e Innovación Docente del Profesorado | № 2 (2019) Esta obra se distribuye con la licencia Creative Commons Reconocimiento-NoComercial-SinObraDerivada 
¿RECOMENDARÍAS A TU ORGANIZACIÓN ADOPTAR UNA VISIÓN AMPLIA DEL MERCADO?

Las necesidades

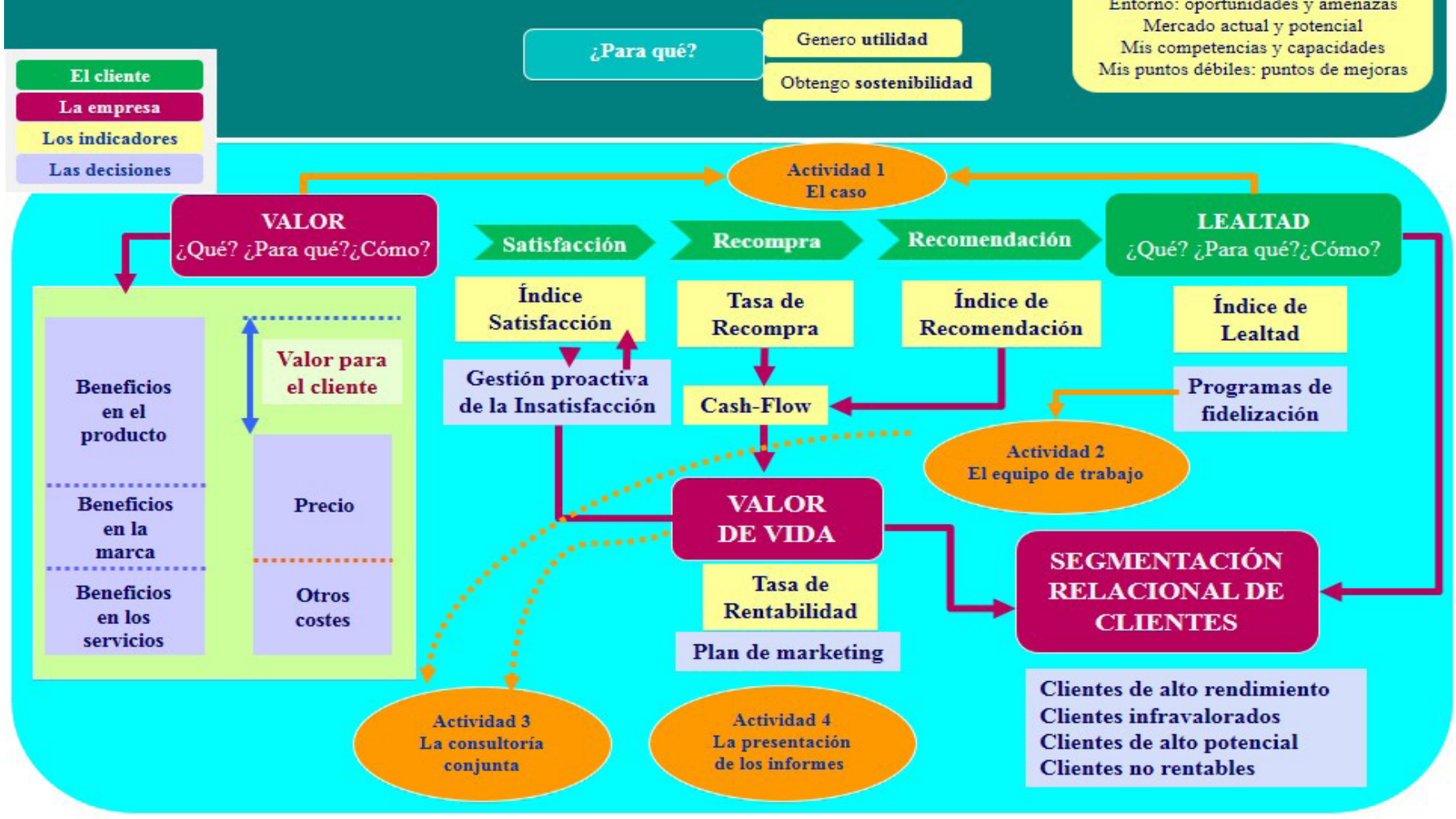

Figura 1. Mapa de contenidos y problemas del Ciclo de Mejora en el Aula.

Jornadas de Formación e Innovación Docente del Profesorado | № 2 (2019)

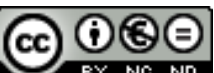
Esta obra se distribuye con la licencia Creative Commons Reconocimiento-NoComercial-SinObraDerivada Internacional (CC BY-NC-ND 4.0.) 
En su elaboración se han seguido los siguientes principios: que el mapa resulte útil (funcional); no necesariamente muy complejo; y que sea comunicable. La reflexión sobre los contenidos me ha llevado a seleccionar los más determinantes, para trabajarlos con profundidad adecuada, y a plantearlos en forma de preguntas "intrigantes" (Bain, 2007). Por ello, el mapa parte de una pregunta inicial, global, que inicia el razonamiento en la temática que se va a abordar: ¿Recomendarías a tu empresa que adopte una visión amplia del mercado? Se trata de una pregunta amplia. Por tanto, dar respuesta a esta cuestión requiere desglosar esa pregunta en una secuencia de preguntas (Finkel, 2008). Las tres cuestiones concretas planteadas dan entrada en el proceso a los contenidos conceptuales, procedimentales y actitudinales. Entre todos ellos, los siguientes adoptan el rol de conceptos estructuradores dentro del proceso: valor, lealtad y valor de vida.

Dentro del mapa, he utilizado colores para situar estos conceptos estructuradores en el nivel o ámbito de la empresa (color granate), o bien dentro del nivel o ámbito del cliente (color verde).

El mapa diseñado indica que la visión amplia del mercado tiene como punto de partida el conocimiento de las necesidades de los clientes, lo que permitirá diseñar productos y servicios adecuados para cubrir esas necesidades de un modo satisfactorio y útil para los clientes, y, al mismo tiempo, rentable para la empresa a largo plazo (sostenibilidad).

El planteamiento realizado genera en los estudiantes la necesidad de disponer de información sobre los conceptos estructuradores mencionados, y también sobre los procedimientos concretos e indicadores necesarios para aplicarlos, medirlos y evaluarlos. Además, la comprensión y asimilación de estos conceptos y procedimientos instalará en ellos actitudes de indagación, de profundizar en 
las necesidades de otros, de promover no sólo los intereses propios e individuales, sino también los de los demás, y a nivel no sólo individual, sino también colectivo.

En un plano más concreto, el planteamiento anterior conduce a analizar y medir (construcción de indicadores) los conceptos de valor, satisfacción, recompra, recomendación, lealtad y valor de vida. La información generada por estos indicadores orientará la toma de decisiones sobre los beneficios y costes que incorporan los productos y servicios financieros, los planes de marketing asociados, los programas de fidelización de los clientes, su segmentación relacional y la gestión proactiva de su posible insatisfacción.

En definitiva, el proceso de aprendizaje de los estudiantes requiere ir comprendiendo de forma progresiva los contenidos señalados, pero no sólo a nivel conceptual. No se trata sólo de conocer, sino de comprender y saber utilizar. Esta ampliación de objetivos permite integrar en el proceso de aprendizaje los contenidos actitudinales, así como los contenidos procedimentales, para así poder alcanzar el nivel de aprendizaje deseado.

En el mapa de contenidos y problemas propuesto se han integrado, asimismo, diversas actividades para alcanzar los objetivos de aprendizaje establecidos.

\section{Cómo enseñar: los principios didácticos y la metodología de enseñanza}

El punto de partida del ciclo es el principio didáctico de mejora progresiva en el proceso de aprendizaje. Por ello, se ha diseñado un conjunto de actividades que incorporan de manera paulatina y acumulativa los objetivos de aprendizaje establecidos, lo que genera a los estudiantes niveles de exigencia y habilidades crecientes (incremento gradual de complejidad). 
Otros principios didácticos tenidos en cuenta son tomar como punto de partida el modelo real de mis estudiantes, su punto de partida y su visión (Giné, 2008), y adaptar el diseño y la aplicación del ciclo de mejora a los recursos disponibles (número de estudiantes que participan en el proceso, tecnología y tiempo disponibles y diseño de espacios).

Por lo que respecta al modelo metodológico, se ha aplicado un modelo investigativo o constructivista centrado en el alumno (De Alba y Porlán, 2017; Delval, 2000), en el que, como docente, asumo un rol motivador y dinamizador, no meramente transmisor, que potencie el aprendizaje de los alumnos de forma activa, implicada, creativa y, en la medida de lo posible, autónoma. Para ello se han utilizado diferentes recursos didácticos, que dan apoyo a las actividades del ciclo de mejora, y que propicien prácticas eficaces (Álvarez, 2016).

Jornadas de Formación e Innovación Docente del Profesorado | № 2 (2019) Esta obra se distribuye con la licencia Creative Commons 


\section{La secuencia de actividades}

Seguidamente se presenta un cuadro resumen de la secuencia de actividades desarrolladas (Tabla 1) y a continuación una breve descripción de dichas actividades.

Tabla 1. Actividades desarrolladas durante el Ciclo de Mejora en el Aula.

\begin{tabular}{|c|c|}
\hline Duración total & 11 horas (del 8 al 30 de abril 2019) \\
\hline Actividades (9 en total) & $\begin{array}{l}\text { Actividad previa: Exploración de conocimien- } \\
\text { tos y capacidades generales de partida. }\end{array}$ \\
\hline $\begin{array}{l}2 \text { actividades de evalua- } \\
\text { ción inicial. }\end{array}$ & $\begin{array}{l}\text { Actividad 1.1: Identificación de los esquemas } \\
\text { iniciales mediante cuestionario (el caso 1). } \\
\text { Actividad 1.2: El debate (del caso) y el pro- } \\
\text { ceso de aprendizaje teórico-práctico de los } \\
\text { conceptos clave. } \\
\text { Actividad 2: El trabajo de equipo (creación de } \\
\text { indicadores). }\end{array}$ \\
\hline $\begin{array}{l}6 \text { actividades para el } \\
\text { aprendizaje. }\end{array}$ & $\begin{array}{l}\text { Actividad 3.1: La consultoría conjunta sobre } \\
\text { valor de vida. } \\
\text { Actividad 3.2: La consultoría conjunta sobre } \\
\text { segmentación relacional (caso 2). } \\
\text { Actividad 4: Las toma decisiones y la elabo- } \\
\text { ración de informes finales. } \\
\text { Actividad 5: La presentación de los informes. }\end{array}$ \\
\hline $\begin{array}{l}1 \text { actividad de evaluación } \\
\text { final }\end{array}$ & $\begin{array}{l}\text { Actividad 6: El caso } 3 \text { y los modelos mentales } \\
\text { finales de los estudiantes. }\end{array}$ \\
\hline
\end{tabular}

Actividad previa: Exploración de conocimientos y capacidades generales de partida

El sentido educativo de esta actividad es identificar los conocimientos y capacidades previos que tienen los estudiantes acerca de conceptos e instrumentos que deberían 
haber adquirido en otras asignaturas ya cursadas y en sus distintas etapas formativas previas a la universitaria. De este modo, se pueden detectar conceptos previos poco consolidados entre los estudiantes, y que pueden representar obstáculos en el aprendizaje de los conceptos estructuradores (Grupo de alto nivel para la modernización de la enseñanza superior de la UE, 2013). Para ello utilizo un cuestionario inicial basado en un caso práctico que se enmarca en el contexto de la atención a clientes financieros.

Actividad 1.1. Identificación de los esquemas iniciales mediante cuestionario

Esta actividad tiene como finalidad aproximarnos al conocimiento de las ideas iniciales de los estudiantes que están vinculadas a los conceptos estructuradores del tema (Rivero y Porlán, 2017). Para ello se ha diseñado un caso que responda a una situación real con la que los estudiantes puedan identificarse fácilmente. El cuestionario se realiza de forma individual para poder establecer escaleras individuales de aprendizaje.

Actividad 1.2. El debate y el proceso de aprendizaje teórico-práctico de los conceptos clave

Para optimizar el tiempo disponible se ha vinculado esta actividad al caso diseñado para evaluar los modelos de partida de los estudiantes, y así obtener efectos sinérgicos. Se desarrolla en forma de debate basado en un guion previamente diseñado, que sirve como base para la labor de equipo y el análisis de los conceptos estructuradores del tema.

Actividad 2. El trabajo en equipo

Los estudiantes trabajan en grupos de 3-4 integrantes, y su labor está apoyada y supervisada por mí. Podrán utilizar los dispositivos electrónicos que aporten. Parto del principio de que los equipos de trabajo potencian y 
generan inteligencia colectiva. El objetivo es que los alumnos pongan en práctica los contenidos conceptuales y actitudinales asociados a los conceptos abordados en la clase anterior, creando para ello indicadores. Por tanto, esta segunda actividad se centra en el desarrollo de contenidos procedimentales. He diseñado un guion que permita a los equipos de trabajo proceder de una manera organizada y eficiente, con sugerencias tales como analizar indicadores ya existentes en el texto, buscar otros ejemplos en los que se apliquen y localizar información para elaborar nuevos indicadores.

\section{Actividad 3.1. La consultoría conjunta} sobre el valor de vida

Los alumnos abordan el concepto de valor de vida a nivel conceptual y procedimental de manera simultánea, pues es la mejor vía de comprensión y asimilación de este concepto estructurador. Para ello, se diseña un proceso para analizar el valor de vida del cliente tanto a nivel cuantitativo como a nivel cualitativo. En mi sondeo inicial sobre el conocimiento de conceptos globales del alumnado he comprobado que los estudiantes parten de una percepción bastante clara sobre la valoración de inversiones, lo cual debe facilitar considerablemente el análisis cuantitativo. Centraremos por tanto la atención especialmente en el análisis cualitativo.

He seleccionado un enunciado "impactante" que genere una intriga/debate en mis estudiantes (Mora, 2013) a modo de "taller conceptual" (Finkel, 2008): “¿Qué hacer cuando un cliente deja de ser rentable?". Utilizaré los diferentes puntos de vista que se pongan de manifiesto para abordar el concepto y la medida del valor de vida del cliente. Tengo preparados diversos ejemplos prácticos reales. Esta actividad se realizará de manera conjunta en clase. 
Actividad 3.2. La consultoría conjunta

sobre segmentación relacional

Esta segunda actividad dentro de la consultoría conjunta introduce el último concepto estructurador del tema: la segmentación relacional. Los resultados del cuestionario inicial indicaban que el concepto y las técnicas de segmentación están menos consolidados entre mis alumnos. Es, por tanto, la tarea más densa y exigente que van a abordar los estudiantes, y la que les requerirá mayor grado de dedicación y creatividad fundamentada (Bain, 2007). He seleccionado un caso real de segmentación del mercado de una entidad financiera (disponible en: http:/ / amint.es/pdf/es/casos-exito/SEGMENTACION_BANCA.pdf, que se proyecta en clase, con un debate posterior, basado en un guion que he diseñado para orientar la consulta y ganar eficiencia.

Actividad 4. La toma de decisiones y la elaboración de informes finales

En esta actividad los estudiantes tendrán que realizar y justificar una segmentación relacional de clientes en un caso real que deberán proponer y defender. Se produce así el máximo nivel de integración de contenidos conceptuales, procedimentales y actitudinales.

Actividad 5. La presentación de los informes

Los equipos de trabajo presentarán sus informes en el formato y tiempo establecidos y se extraerán conclusiones finales (manejando conceptos, procedimientos y actitudes).

Actividad 6. El caso 3 y los modelos

mentales finales de los estudiantes

El sentido educativo de esta actividad es conocer los modelos mentales que han alcanzado mis estudiantes 
-sobre todo en el dominio conceptual- tras aplicar el ciclo de mejora que he diseñado. Esta información servirá de base para evaluar el ciclo de mejora aplicado y para diseñar mejoras futuras.

Los estudiantes responderán a preguntas vinculadas a un caso práctico que he diseñado específicamente para esta actividad, enmarcado en el sector bancario, y más concretamente, en el producto hipotecario. Las cuestiones planteadas son equivalentes a las establecidas en el caso 1 , con la finalidad de comparar los modelos mentales iniciales y finales de los estudiantes y, en consecuencia, el grado de aprendizaje logrado.

\section{Aplicación del Ciclo de Mejora en el Aula}

El desarrollo de las actividades diseñadas ha generado informaciones y reflexiones muy interesantes. Seguidamente se destacan las fundamentales.

Actividad 1.1. Identificación de los esquemas iniciales mediante cuestionario

Cada estudiante ha tenido a su disposición de forma individualizada el enunciado del caso, lo que les ha permitido poder consultarlo y utilizarlo en el desarrollo de esta actividad. Para llevar a cabo mi labor gestora y conductora del aprendizaje, he utilizado un guion que diseñé para orientar el debate, cuyos puntos representan los objetivos de aprendizaje de la actividad. Al analizar los datos obtenidos, he podido obtener informaciones que me han llevado a realizar algunos reajustes tanto en el mapa de contenidos como en algunas de las actividades diseñadas (Rivero y Porlán, 2017). 
Actividad 1.2. El debate y el proceso de aprendizaje teórico-práctico de los conceptos clave

Hemos organizado la información en la pizarra, usando para ello 4 zonas: 1 a. Problema fundamental que se ha producido (Escribano y Del Valle, 2008); 2a. Principales consecuencias; 3a. Soluciones; 4a. Cómo evitarlo. De esta labor se han ocupado, de forma voluntaria, un alumno y una alumna.

Tras finalizar el debate inicial, se ha realizado una síntesis de ideas para obtener una visión de conjunto. A ello he unido la proyección de imágenes, un vídeo y varios links.

Esta primera actividad del ciclo de mejora me ha permitido observar y entender el punto de partida de mis estudiantes en la temática abordada. Me ha resultado curioso e interesante comprobar que algunos de ellos se han sentido identificados en el rol de clientes, mientras que otros han asumido más la perspectiva de la empresa. Es precisamente esta doble perspectiva la que permite obtener una mejor visión y comprensión de las situaciones planteadas. También he corroborado que asignarles roles ha aumentado la motivación y la proactividad.

El tiempo restante de esta primera clase del ciclo de mejora se ha dedicado a trabajar en una primera aproximación a los conceptos claves del tema. Para ello hemos usado la clase general en formato interactivo, planteando nuevas preguntas y proporcionando ejemplos reales en formato digital que he preparado previamente (noticias en prensa y redes sociales, proyección de un vídeo, imágenes gráficas). Hemos dedicado los 5 minutos finales de la clase a sintetizar y afianzar las ideas más interesantes y los conceptos estructuradores abordados.

Jornadas de Formación e Innovación Docente del Profesorado | № 2 (2019) Esta obra se distribuye con la licencia Creative Commons 
Los estudiantes han trabajado en 10 grupos de 3-4 integrantes, y su labor ha sido apoyada y supervisada por mí. Han podido utilizar los dispositivos electrónicos que han aportado. Parto del principio de que los equipos de trabajo potencian y generan inteligencia colectiva. Antes de iniciar su labor, he proporcionado a los equipos de trabajo el guion que previamente diseñé para esta actividad, de manera que aumentasen su organización y eficiencia (Finkel, 2008).

Cada equipo de trabajo ha desarrollado su búsqueda de información basada no sólo en la bibliografía general que aparece en el programa de la asignatura, sino también mediante bibliografía interactiva y recursos electrónicos que han traído ese día al aula. A partir de la información recogida, cada grupo ha generado un debate y valoración internos, y han preparado un breve informe. Durante esta clase, mi misión ha consistido en interactuar de forma personalizada con cada grupo para conocer cómo estaban desarrollando su actividad, orientarles y ayudarles a enfocar y abordar problemas o dudas. Y he ido realizando anotaciones en mi diario de clase, lo que parece haber aumentado la motivación de los estudiantes. Destaco en mis observaciones que los grupos que han desplegado comportamientos más eficaces y avanzados son los que han partido de una buena organización, asignación de roles a cada miembro del grupo, planificación temporal de cada tarea, coordinación y tolerancia a la diversidad de ideas.

Hemos dedicado los 5 últimos minutos de la sesión a sintetizar ideas, realizar sugerencias de mejora de la actividad y asignación de las tareas de elaboración de un dossier resumen que pondremos a disposición de todos los estudiantes en la plataforma de enseñanza virtual. 
El caso les ha resultado bastante interesante, aunque quizás un poco profundo para el nivel básico del que muchos partían. Sin embargo, los estudiantes más avanzados en este concepto han ido impulsando la integración de los más rezagados, ayudados por mí y por el guion secuencial que he diseñado (me ha resultado muy útil). La sinergia ha sido bastante interesante, superándose el miedo inicial al error (Astolfi, 1999).

Poco a poco hemos avanzado desde el análisis conceptual hasta el procedimental y el actitudinal. Es el procedimental el que ha presentado más dificultad, por lo que he decidido simplificar los objetivos de aprendizaje que había establecido inicialmente.

El análisis y el aprendizaje generados mediante esta actividad me han llevado a proponer a mis estudiantes las dos últimas actividades del ciclo de mejora. Para ello, hemos dedicado los últimos minutos de la clase a organizar de nuevo grupos de entre 3 y 4 participantes. Su misión inicial ha consistido ahora en seleccionar un caso real de segmentación que deberán aportar en la siguiente clase (actividad de búsqueda independiente, fuera del aula).

Actividad 4. La toma de decisiones y la elaboración de informes finales

Los alumnos han acudido al aula con su propuesta de partida: el caso de segmentación real que han seleccionado. Cada grupo ha asignado_roles a cada integrante; los más habituales han sido coordinación, búsqueda de información adicional y toma de notas. Tras generar un debate y valoración internos, cada grupo ha elaborado un informe. Durante esta actividad, mi rol ha consistido de nuevo en atender de forma personalizada a cada grupo para conocer cómo están desarrollando su actividad, orientarles y ayudarles a enfocar y abordar problemas o dudas, guiándome por la idea de reducir mi actuación a 
lo estrictamente necesario, para que sean los estudiantes quienes asuman el rol central en la actividad.

Actividad 5. La presentación de los informes

Cada equipo de trabajo ha realizado su exposición durante un máximo de 15 minutos. Dado que previamente les expliqué la importancia de respetar estos tiempos, no he tenido necesidad de interrumpir la intervención de ningún grupo. Al finalizar hemos realizado un balance final.

Actividad 6. El caso 3 y los modelos mentales finales de los estudiantes

Una vez desarrolladas las distintas actividades que componen el Ciclo de Mejora en el Aula, resulta conveniente valorar el impacto que han tenido en el aprendizaje de los estudiantes. Esto requiere diseñar una escala de medida que permita comparar el nivel inicial de partida y el nivel finalmente alcanzado. Por ello, para cada uno de los conceptos estructuradores analizados se han definido cuatro niveles: nivel 1: visión muy limitada; nivel 2: visión simple pero orientada; nivel 3: visión amplia; nivel 4: visión amplia + creativa. Seguidamente se presenta los principales resultados alcanzados para los conceptos clave del tema, utilizando para ello las escaleras de aprendizaje ( $\mathrm{Fi}$ guras 2, 3, 4 y 5).

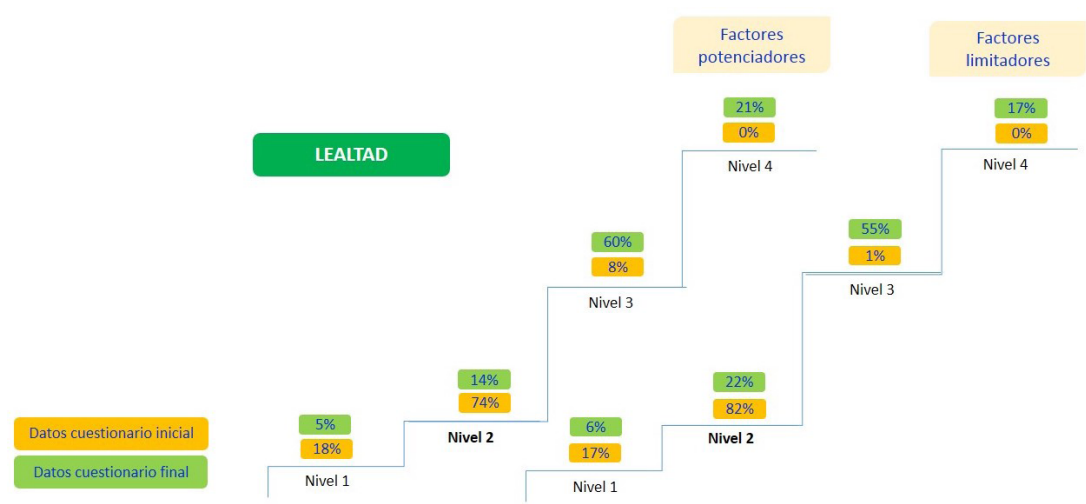

Figura 2. Escaleras de aprendizaje del concepto "lealtad".

Jornadas de Formación e Innovación Docente del Profesorado | № 2 (2019) Esta obra se distribuye con la licencia Creative Commons Reconocimiento-NoComercial-SinObraDerivada 
Conclusión: La mayoría de los estudiantes parten del nivel 2 con respecto a los factores que potencian y limitan la lealtad del cliente. Tras aplicar el ciclo de mejora esa mayoría se traslada claramente al nivel 3 , e incluso en torno a un 20\% logran el máximo nivel en la escala.

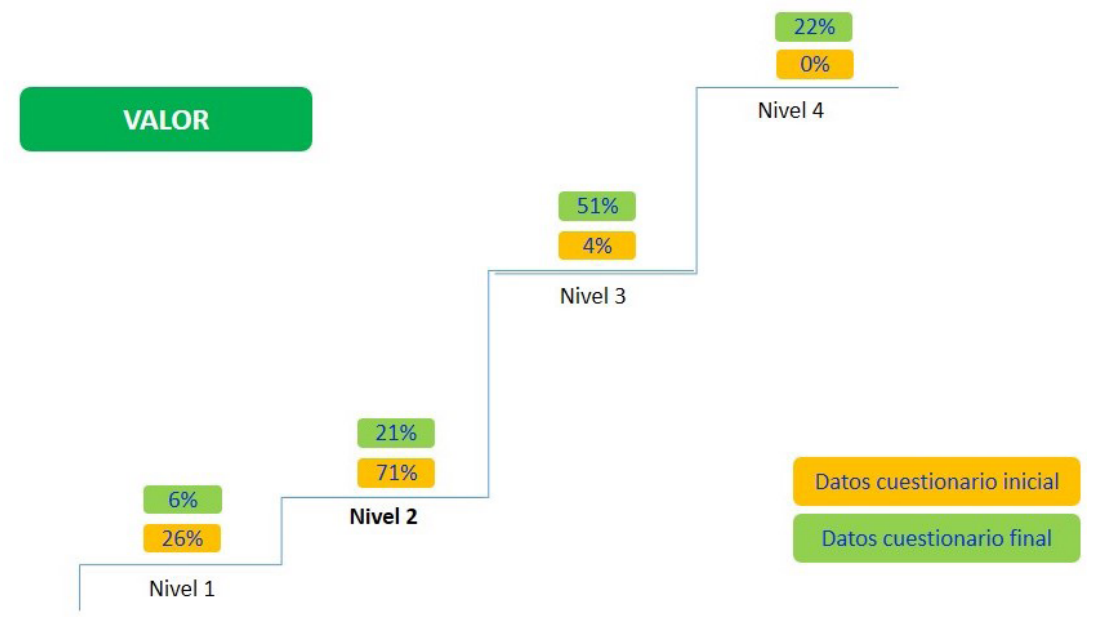

Figura 3. Escaleras de aprendizaje del concepto "valor".

Conclusión: La mayoría de los estudiantes parten del nivel 1 y 2 con respecto al concepto de valor percibido por el cliente. Tras aplicar el ciclo de mejora, esa mayoría se traslada claramente al nivel 3, e incluso el 22\% logran el máximo nivel en la escala.

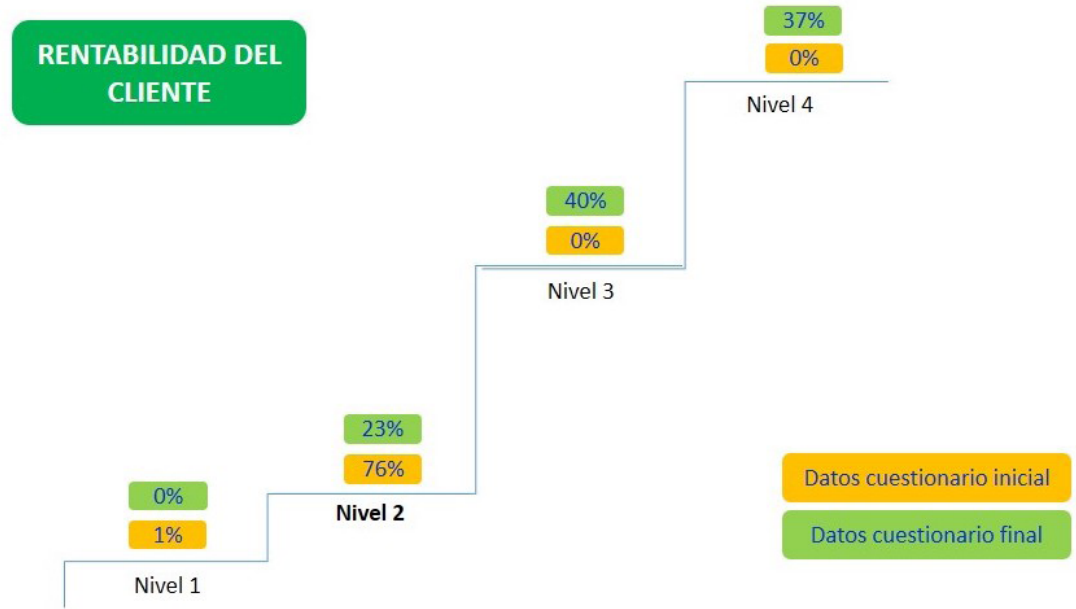

Figura 4. Escaleras de aprendizaje del concepto "rentabilidad".

Jornadas de Formación e Innovación Docente del Profesorado | № 2 (2019) Esta obra se distribuye con la licencia Creative Commons Reconocimiento-NoComercial-SinObraDerivada 4.0 Internacional (CC BY-NC-ND 4.0.) 
Conclusión: La mayoría de los estudiantes parten del nivel 2, lo cual era esperable, pues la rentabilidad es un concepto con un claro componente financiero. No obstante, inicialmente sólo el 23\% de los estudiantes perciben en dicho concepto algún matiz comercial. Tras aplicar el ciclo de mejora, el $77 \%$ de los estudiantes se sitúan en los niveles 3 y 4 de la escalera de aprendizaje, y ninguno queda situado en el nivel inicial.

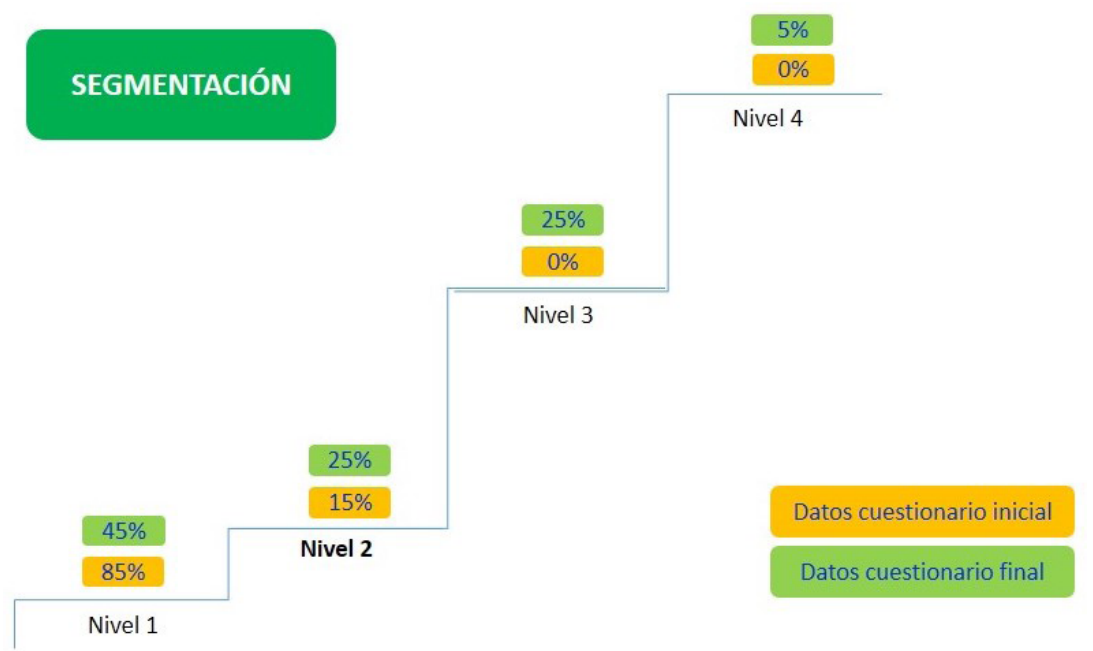

Figura 5. Escaleras de aprendizaje del concepto "segmentación”.

\section{Evaluación del Ciclo de Mejora Docente}

\section{Las dificultades}

El ciclo de mejora llevado a cabo ha resultado exigente, pues el número de actividades puestas en marcha ha sido elevado. Ha sido precisamente el tiempo el "enemigo" más directo. Pero, de no haber sido así, no habría podido comprobar muchas cosas: avances, obstáculos, 
nuevos elementos estimuladores para mis estudiantes, circunstancias del entorno que ayudan y que limitan, influencia de factores como el momento del curso, e incluso las temperaturas.

Otra de las principales dificultades que he tenido que afrontar es el elevado número de alumnos del grupo. Crear un escenario de aprendizaje adecuado en estas condiciones es bastante más complicado que en grupos reducidos. Finalmente, destaco que los recursos fisicos y tecnológicos del aula no han favorecido la aplicación del ciclo de mejora; especialmente el escenario estático con la disposición lineal y fija de las bancas de asientos.

\section{Evaluación del aprendizaje individual de los estudiantes}

Uno de los aprendizajes más valiosos que he obtenido en esta experiencia es la vinculación de la evaluación de los estudiantes con el análisis de la evolución de sus ideas (Cano y Barrios, 2011; Rivero y Porlán, 2017). Por ello, considero muy interesante la elaboración de gráficos que nos ayuden a visualizar y, por tanto, a hacer tangible esa evolución. En el caso de este ciclo de mejora, en el cuestionario inicial participaron 49 estudiantes, y en el cuestionario final fueron 42 estudiantes. Sobre el aprendizaje de los conceptos estructuradores básicos ya he presentado, anteriormente, los resultados globales. Me centro ahora en la evolución del aprendizaje de los estudiantes desde la perspectiva individual. Para poder valorar esa evolución, he seleccionado 5 estudiantes -identificados- que realizaron tanto el cuestionario inicial como el final: dos que partiesen de nivel 1, dos que partiesen de nivel 2, y uno partiese de nivel 3 (dado que es el nivel de partida menos frecuente). Ningún estudiante partía de nivel 4 en ninguna de las preguntas planteadas en los cuestionarios. He representado los resultados en la Tabla 2, en la que se 
recogen los niveles en las respuestas a los cuestionarios inicial y final, así como el ascenso o permanencia en la evolución del aprendizaje de un cuestionario a otro, mediante flechas.

Tabla 2. Resultados de la evolución del aprendizaje de una muestra de estudiantes.

\begin{tabular}{|c|c|c|c|c|c|c|c|c|c|c|c|c|c|c|c|}
\hline \multirow[t]{2}{*}{$\mathbf{E}$} & \multicolumn{3}{|c|}{$\begin{array}{c}\text { Pregunta } 1 \\
\text { Lealtad }\end{array}$} & \multicolumn{3}{|c|}{$\begin{array}{l}\text { Pregunta } 2 \\
\text { Valor }\end{array}$} & \multicolumn{3}{|c|}{$\begin{array}{c}\text { Pregunta } 3 \\
\text { Rentabilidad }\end{array}$} & \multicolumn{3}{|c|}{$\begin{array}{l}\text { Pregunta } 4 \\
\text { Herramien- } \\
\text { tas Lealtad }\end{array}$} & \multicolumn{3}{|c|}{$\begin{array}{c}\text { Pregunta } 5 \\
\text { Herramientas } \\
\text { Segmentación }\end{array}$} \\
\hline & Cl & CF & EF & $\mathrm{Cl}$ & CF & EF & Cl & CF & EF & $\mathrm{Cl}$ & CF & EF & $\mathrm{Cl}$ & CF & EF \\
\hline E1 & 1 & 2 & $\uparrow$ & 1 & 3 & $\uparrow$ & 1 & 3 & $\uparrow$ & 1 & 2 & $\uparrow$ & 1 & 2 & $\uparrow$ \\
\hline E2 & 1 & 3 & $\uparrow$ & 1 & 2 & $\uparrow$ & 1 & 3 & $\uparrow$ & 1 & 2 & $\uparrow$ & 1 & 2 & $\uparrow$ \\
\hline E3 & 2 & 3 & $\uparrow$ & 2 & 4 & $\uparrow$ & 2 & 2 & $\leftrightarrow$ & 2 & 3 & $\uparrow$ & 2 & 2 & $\leftrightarrow$ \\
\hline E4 & 2 & 4 & $\uparrow$ & 2 & 3 & $\uparrow$ & 2 & 4 & $\uparrow$ & 2 & 3 & $\uparrow$ & 2 & 3 & $\uparrow$ \\
\hline E5 & 3 & 4 & $\uparrow$ & 3 & 3 & $\leftrightarrow$ & 3 & 3 & $\leftrightarrow$ & 3 & 3 & $\leftrightarrow$ & * & * & * \\
\hline
\end{tabular}

Como puede observarse, en general, la evolución ha sido favorable. Compruebo, al igual que en el análisis global de los datos, que las mayores dificultades están relacionadas con los contenidos procedimentales más comerciales, en concreto, las herramientas de lealtad y segmentación. Serán, pues, estas áreas las que más atención didáctica necesiten en el futuro.

En términos globales, considero que la aplicación de este ciclo de mejora ha sido bastante interesante, que nos ha implicado tanto a mis estudiantes como a mí misma, y que ha ayudado a que muchos de ellos alcancen niveles de aprendizaje elevados (niveles 3 y 4). 
Al finalizar el ciclo de mejora, he solicitado a mis estudiantes una valoración de la experiencia didáctica, en una escala de 1 a 5 puntos; la valoración media se ha situado en 4.3 puntos sobre un máximo de 5 puntos, por lo que su balance global ha sido muy positivo, lo que refuerza mi satisfacción.

\section{Los aspectos que voy a mantener}

El aprendizaje que he podido desarrollar a lo largo de esta experiencia de ciclo de mejora me ha aportado numerosas ideas, que resumo en los siguientes principios (Bain, 2007): 1. Crear un entorno para el aprendizaje crítico natural; 2. Conseguir la atención del estudiante y no perderla; 3. Comenzar con los estudiantes en lugar de con la disciplina; 4. Buscar compromisos; 5. Ayudar a los estudiantes a aprender fuera de clase; 6 . Atraer a los estudiantes al razonamiento disciplinar; 7 . Crear experiencias de aprendizaje diversas. Se trata de activar a mis estudiantes, de fomentar que se comprometan con su propio aprendizaje, y que visualicen las actividades como una labor de equipo, en la que el rol de sus compañeros va más allá del simple "acompañamiento pasivo a mis explicaciones", y en la que mi rol va más allá de la transmisión unidireccional de datos y la evaluación de lo que ellos hayan conseguido memorizar.

Destaco también algo que ha funcionado muy bien: ha habido un incentivo conocido desde el principio por los estudiantes, el hecho de que voy a valorar su participación activa y su implicación en este ciclo de mejora -que es algo que les propuse desde el principio como "acción voluntaria". Espero que su motivación interna haya crecido a lo largo de este proceso, pero creo que añadirle motivación externa es una buena mezcla. 
A ello hay que unir que les expliqué en qué consistía el ciclo de mejora, por qué me implicaba en esto, el esfuerzo que me suponía y mi absoluta necesidad de su colaboración para llegar a buen puerto. Y ellos me han respondido. En definitiva, el diseño y la puesta en práctica de este ciclo de mejora me han permitido seguir pensando y reflexionando sobre mi docencia. Y me ha generado nuevas ideas para consolidar caminos ya iniciados, y emprender otros.

Igualmente destaco la adopción de una premisa fundamental: para llegar a un objetivo, necesitamos conocer de dónde partimos. De ahí la absoluta necesidad de conocer los esquemas mentales o modelos iniciales de nuestros estudiantes, su diversidad y sus posibles agrupaciones en patrones comunes. También considero muy importante diseñar un instrumento de recogida de información inicial y final basado en preguntas abiertas (Rivero y Porlán, 2017). Me decanto por el cuestionario, pues el número de estudiantes con los que trabajo es bastante elevado y sus respuestas escritas es información permanente que puedo evaluar de forma más objetiva y progresiva. Además, me resulta muy interesante y aplicable la escalera de aprendizaje complementada con actividades tanto formativas como de contraste.

Finalmente, destaco algunos aspectos cualitativos imprescindibles y muy valiosos:

- Proceso de investigación y de mejora continua (Rué, 2007).

- Implicación y participación de profesores y de estudiantes (Giné, 2009).

- Motivaciones no sólo extrínsecas, también intrínsecas.

- Evaluación formativa (Rivero y Porlán, 2017).

- Para mejorar y aprender hay que conocer y superar obstáculos. 


\section{Referencias bibliográficas}

Álvarez Teruel, J. D. (Coord.) (2016). Innovaciones metodológicas en docencia universitaria: resultados de investigación. Alicante: ICE de la Universidad de Alicante.

Astolfi, J. P. (1999). El "error", un medio para enseñar. Sevilla: Díada.

Bain, K. (2007). Lo que hacen los mejores profesores universitarios. Valencia: Publicacions de la Universitat de València.

Cano García, E.; Barrios, R. (2011). Buenas prácticas en la evaluación de competencias: cinco casos de Educación Superior. Barcelona: Laertes.

De Alba, N. y Porlán, R. (2017). La metodología de enseñanza. En R. Porlán (Coord.), Enseñanza Universitaria. Cómo mejorarla (pp. 37-54). Madrid: Morata.

Delval, J. (2000). Aprender en la vida y en la escuela. Madrid: Morata.

Escribano, A. y Del Valle, A. (2008). El aprendizaje basado en problemas. Una propuesta metodológica en Educación Superior. Madrid: Narcea.

Finkel, D. (2008). Dar clase con la boca cerrada. Valencia. Publicacions de la Universitat de València.

Giné Freixes, N. (2009). Cómo mejorar la docencia universitaria: El punto de vista del estudiantado. Revista Complutense de Educación, 20(1), 117-134.

Grupo de alto nivel para la modernización de la enseñanza superior de la UE (2013). Enseñar a los profesores a enseñar. Recomendaciones para la mejora de la calidad de la enseñanza y el aprendizaje en la enseñanza superior. Comisión Europea. Recuperado de http://europa.eu/rapid/press-release_IP-13-554_es.htm

Mora, F. (2013). Neuroeducación. Madrid: Alianza.

Rivero, A. y Porlán, R. (2017). La evaluación de la enseñanza universitaria. En R. Porlán (Coord.), Enseñanza Universitaria. Cómo mejorarla (pp. 73-92). Madrid: Morata.

Rué, J. (2007). Enseñar en la Universidad. El EEES como reto para la educación. Madrid: Narcea.

Jornadas de Formación e Innovación Docente del Profesorado | № 2 (2019) Esta obra se distribuye con la licencia Creative Commons 